\title{
Exploring local disorder in fast oxygen ion conductors by atomic pair distribution function analysis
}

\author{
Michela Brunelli ${ }^{1, \mathrm{a}}$ and Monica Ceretti ${ }^{2}$ \\ ${ }^{1}$ Swiss-Norwegian Beamlines, ESRF, BP. 220, 38043 Grenoble, France \\ ${ }^{2}$ Institut de Chimie Moléculaire et des Matériaux ICGM, UMR 5253 CNRS-Université de Montpellier, \\ Place Eugène Bataillon, 34095 Montpellier Cedex 5, France
}

\begin{abstract}
A preliminary structural investigation of local disorder in fully oxidized $\mathrm{La}_{2} \mathrm{NiO}_{4+\delta}$ was performed by Pair Distribution Function (PDF) analyses of X-ray and neutron powder diffraction data. X-ray diffraction data were recorded in-situ on warming from room temperature up to $450^{\circ} \mathrm{C}$.
\end{abstract}

\section{Introduction}

Mixed-valence Ruddlesden-Popper type-oxides allow to take up oxygen ions on interstitial lattice sites, allowing to modify importantly physical properties. An important example is stoichiometric and semiconducting $\mathrm{La}_{2} \mathrm{CuO}_{4.0}$, which can be transformed into a superconductor with a $\mathrm{T}_{c}$ of $42 \mathrm{~K}$ when intercalated with oxygen, up to a stoichiometry corresponding to $\mathrm{La}_{2} \mathrm{CuO}_{4.07}$ [1]. Other phases exist where the extra oxygen stoichiometry can attain important values as is the case for $(\mathrm{Pr} / \mathrm{Nd})_{2} \mathrm{NiO}_{4+\delta}$, or $\mathrm{La}_{2} \mathrm{CoO}_{4+\delta}$, with $0 \leq \delta \leq 0.25$, while the transition metal changes its valence state from pure $2+$ to a mixed valence state of equally $2+/ 3+$. The uptake of oxygen ions plays also a crucial role for low temperature oxygen mobility as has been shown recently in a combination of inelastic neutron scattering studies together with DFT-based simulations for $\mathrm{Nd}_{2} \mathrm{NiO}_{4+\delta}$ [2]. The main outcome of these investigations was that oxygen mobility has been simulated to take place already at ambient temperature in a time interval of some $p s$. For $\mathrm{La}_{2} \mathrm{CuO}_{4.07}$ a combination of single crystal neutron diffraction together with lattice dynamical simulations have evidenced that the apical oxygen atoms are localized at $20 \mathrm{~K}$, while they move dynamically at ambient temperature on a circle of about $1 \AA$ around their equilibrium positions [3], equally showing libration modes in the [110] direction of the F-cell, i.e. towards the interstitial sites. Strong librations towards [110] are forced by the presence of interstitial oxygen atoms, rendering their local environment strongly disordered. Since the presence of interstitial oxygen atoms force the apical oxygen atoms to leave their equilibrium conditions, their structural description is complex and appears to be anharmonic. This structural complexity and apical oxygen disorder is difficult to analyze by classical diffraction methods. Our approach here is therefore to explore the real structure by PDF analysis on a highly oxygen doped $\mathrm{K}_{2} \mathrm{NiF}_{4}$-type oxide and to investigate especially any kind

\footnotetext{
${ }^{a}$ Corresponding author: brunelli@esrf .fr
}

This is an Open Access article distributed under the terms of the Creative Commons Attribution License 4.0, which permits unrestricted use, distribution, and reproduction in any medium, provided the original work is properly cited. 

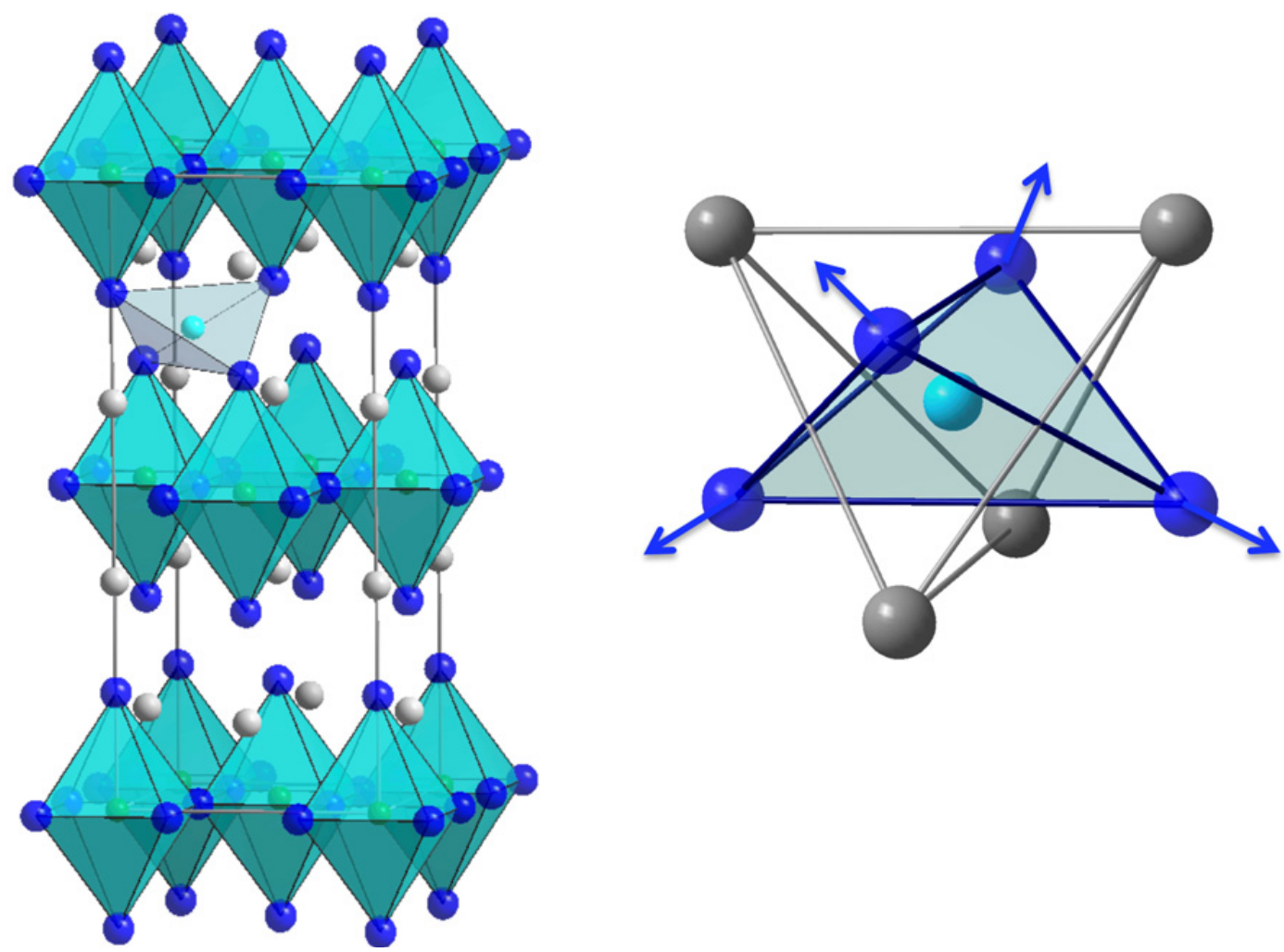

Figure 1. Oxygen intercalation into $\mathrm{K}_{2} \mathrm{NiF}_{4}$ type oxide frameworks goes along with the occupation of interstitial lattice sites inside the rock salt $\mathrm{RE}_{2} \mathrm{O}_{2}$-layer, while $\mathrm{O}_{\text {int }}$ is tetrahedrally coordinated by $\mathrm{O}_{\text {ap }}$ and $\mathrm{RE}$ atoms. Locally, the oxygen intercalation leads to strong deformation of the tetrahedra, related to a strong increase of the $\mathrm{O}_{\text {int }}-\mathrm{O}_{\text {ap }}$ distance from $2.15 \AA$ to about $2.7 \AA$ [8]. This implies an important symmetrical increase of all intercalated $\mathrm{O}_{\text {int }}\left(\mathrm{O}_{\text {ap }}\right)_{4}$ tetrahedra, pushing implied Oap atoms along the [110] direction of the F-cell towards adjacent interstitial lattice sites. The presence of $\mathrm{O}_{\text {int }}$ is thus crucial for a dynamical activation of the $\mathrm{O}_{\text {ap }}$ to move between the apical and interstitial sites on a shallow energy diffusion pathway.

of oxygen displacements. $\mathrm{La}_{2} \mathrm{NiO}_{4}$ is one special candidate as it combines two important points: a sufficiently high maximum extra oxygen stoichiometry being $\mathrm{La}_{2} \mathrm{NiO}_{4.18}$ combined with a tetragonal lattice symmetry. Since all homologous phases like $(\mathrm{Pr} / \mathrm{Nd})_{2} \mathrm{NiO}_{4+\delta}$ as well as $\mathrm{La}_{2}(\mathrm{Cu} / \mathrm{Co}) \mathrm{O}_{4+\delta}$ turn out to show all orthorhombic symmetry even up to $\delta=0.25$, the tetragonal symmetry of $\mathrm{La}_{2} \mathrm{NiO}_{4.18}$ appears to be much more promising than orthorhombic symmetry in terms of a simplification for possible peak overlapping by powder diffraction experiments and related data analysis.

The structure of $\mathrm{La}_{2} \mathrm{NiO}_{4}$ is schematically outlined in Fig. 1, also showing the interstitial position of intercalated oxygen. Taking into account the average high temperature tetragonal structure in $I 4 / \mathrm{mmm}$ (Eq. to $\mathrm{F} 4 / \mathrm{mmm}$ ), all $\mathrm{NiO}_{6}$-octahedra are perfectly aligned with the c-axis. The structure can be understood as a layer sequence of $\mathrm{NiO}_{6}$-octahedra or alternatively as an intergrowth $\mathrm{NiO}_{2-}$ sheets alternating with rock salt arranged $\mathrm{La}_{2} \mathrm{O}_{2}$ layers. Oxygen mobility in $\mathrm{La}_{2} \mathrm{NiO}_{4+\delta}$ [4] and $(\mathrm{Pr} / \mathrm{Nd})_{2} \mathrm{NiO}_{4+\delta}$ [5] has been shown to be extremely anisotropic, while the diffusion in the (a,b)-plane is about 3 orders of magnitude higher, compared to a diffusion along the c-axis. The interstitial oxygen atoms are supposed to play an important role for the diffusion mechanism. This can be understood in a way that the deformations induced to the presence of interstitial oxygen atoms lead to important local distortions of the $\mathrm{O}_{\text {int }}\left(\mathrm{O}_{\text {ap }}\right)_{4}$ tetrahedra, pushing all adjacent apical oxygen atoms away from their 
Table 1. Crystallographic data of $\mathrm{La}_{2} \mathrm{NiO}_{4.15(2)}$ obtained at ambient by single crystal neutron diffraction of an as grown crystal in $I 4 / \mathrm{mmm}$. Data collection were obtained on $5 \mathrm{C} 2$ at the hot source of the ORPHEE reactor up to $\sin \Theta / \lambda=1.05 \AA^{-1}$. While the occupancies of the metal atoms were fixed to the ideal values, the oxygen occupancies were refined. It becomes evident that the equatorial occupancies correspond to the expected values, while the position of the apical oxygen atom was split into $(0,0, z)$ and $(x, 0, z)$, as a result of the presence of the interstitial oxygen atoms, pushing partially $\mathrm{O}_{\mathrm{ap}}$ into the [110]-direction. We note that the amount of $\mathrm{O}_{\text {ap }}(2)$ corresponds to almost four times the amount of the $\mathrm{O}_{\text {int }}$, going along with the idea of a symmetrical increase of the $\mathrm{O}_{\text {int }}\left(\mathrm{O}_{\mathrm{ap}}\right)_{4}$ tetrahedra. $U_{i i}$ are in $\AA^{2}$ units.

\begin{tabular}{c|ccccccc}
\hline atom & $\boldsymbol{x}$ & $\boldsymbol{y}$ & $\boldsymbol{z}$ & $\boldsymbol{U}_{\mathbf{1 1}}$ & $\boldsymbol{U}_{\mathbf{2 2}}$ & $\boldsymbol{U}_{\mathbf{3 3}}$ & sof \\
\hline$L a$ & 0 & 0 & $0.36078(6)$ & $0.0066(2)$ & $0.0066(2)$ & $0.0056(3)$ & 0.125 \\
$N i$ & 0 & 0 & 0 & $0.0035(2)$ & $0.0035(2)$ & $0.0106(4)$ & 0.0625 \\
$O_{a p}(1)$ & 0 & 0 & $0.1737(2)$ & $0.017(2)$ & $0.017(2)$ & $0.0074(7)$ & $0.089(5)$ \\
$O_{a p}(2)$ & $0.143(5)$ & 0 & $0.1700(6)$ & $0.006(6)$ & $0.015(3)$ & $0.006(2)$ & $0.039(5)$ \\
$O_{e q}$ & 0.5 & 0 & 0 & $0.0049(4)$ & $0.0078(4)$ & $0.0246(7)$ & $0.126(2)$ \\
$O_{\text {int }}$ & .5 & 0 & 0.25 & $0.010(4)$ & $0.010(4)$ & $0.006(5)$ & $0.0094(14)$ \\
\hline
\end{tabular}

equilibrium positions and along the [100] direction of the $I 4 / \mathrm{mmm}$ cell (Eq. to the [110]-direction of the $\mathrm{F} 4 / \mathrm{mmm}$ cell), i.e. into the direction of neighbouring interstitial lattice sites, which are filled statistically by $2 \%$ only for a stoichiometry corresponding to $\mathrm{La}_{2} \mathrm{NiO}_{4.15}$. The structural disorder is directly seen from the necessity to introduce two $\mathrm{O}_{\text {ap }}$ positions into oxygen intercalated $\mathrm{La}_{2} \mathrm{NiO}_{4+\delta}$ as can be seen in Table 1. The $\mathrm{O}_{\mathrm{ap}}(2)$ with $(x, 0, z)$ coordinates indicates a strong shift into the [100] direction of the I-cell. This then easily leads to a push pull mechanism as described for $\mathrm{La}_{2} \mathrm{NiO}_{4+\delta}$ [6] above $800 \mathrm{~K}$ in the tetragonal phase, as well as for $(\mathrm{Pr} / \mathrm{Nd})_{2} \mathrm{NiO}_{4+\delta}$ [7]. Recent studies, based on a combination of inelastic neutron scattering together with DFT-based Molecular Dynamics simulations and analyzed by a new developed position recurrence method, clearly evidenced for $\mathrm{Nd}_{2} \mathrm{NiO}_{4+\delta}$ that oxygen mobility is activated dynamically as low as ambient temperature [2] allowing exchange between the apical and interstitial oxygen positions on a $p s$ time scale. It becomes thus clear that specific lattice dynamics and related oxygen displacements from their equilibrium positions are a key issue to understand the diffusion mechanisms behind low temperature oxygen mobility. It becomes also clear that classical structure analysis, not taking into account these oxygen librations, which have been shown to be anharmonic, has limitations to evidence from a structural point of view oxygen dynamics and specifically large displacement amplitudes by spherical Gaussian-type ellipsoids. The aim of this study is consequently to take advantage of the additional information PDF analysis can give compared to classical structure refinement, as it takes into account diffuse scattering between the Bragg reflections.

In order to have a highly crystalline $\mathrm{La}_{2} \mathrm{NiO}_{4.18}$, the used polycrystalline sample for PDF analysis was obtained from a ground single crystal, obtained by the floating zone method. Temperature dependent single crystal neutron diffraction analysis have already been investigated by the Maximum Entropy Method [8], showing strong and discrete oxygen displacements at $20 \mathrm{~K}$, while at room temperature and higher librations of the $\mathrm{NiO}_{6}$ octahedra pointing with the apical oxygen atoms towards the interstitial oxygen site became obvious. The main difference comparing single crystal and powder diffraction methods for disordered structures is that discrete intensities of (hkl) are obtained, while total scattering intensities including diffuse one is measured for powder diffraction. We therefore aimed to compare both approaches for $\mathrm{La}_{2} \mathrm{NiO}_{4.18}$, while exploring powder diffraction with neutron and synchrotron radiation.

The different X-ray and neutron scattering lengths (details are given in Table 1) of the elements in this system lead to a complementary structural information.

While traditional crystallography is based on the information content of Bragg scattering reflections and probes only time - and space-averaged structures (average positions, displacement parameters, occupancies), a total scattering method, which includes diffuse and inelastic scattering, gives information about the local instantaneous structure at the nanoscale (e.g. chemical short-range order or local distortions). The Pair Distribution Function (PDF) analysis is the real space analysis of diffraction 
Table 2. Neutron scattering length $b(\mathrm{fm})$, atomic number $Z$ and ionic radius for $\mathrm{La}, \mathrm{Ni}$ and $\mathrm{O}$.

\begin{tabular}{c|ccc}
\hline Element & $\boldsymbol{b} / \boldsymbol{f m}$ & $\boldsymbol{Z}$ & $\boldsymbol{i r} / \boldsymbol{A}$ \\
\hline $\mathrm{La}$ & 8.24 & 57 & 1.03 \\
$\mathrm{Ni}$ & 10.3 & 28 & 0.55 \\
$\mathrm{O}$ & 5.80 & 8 & 1.4 \\
\hline
\end{tabular}

data in which both Bragg and diffuse scattering contributions are considered, and it is a unique tool to determine the local and medium range deviations with respect to an ideally periodic structure $[9,10]$.

The Pair Distribution Function $\mathrm{G}(r)$ is experimentally determined via sine Fourier transform of a properly corrected total scattering function $S(Q)$ :

$$
G(r)=\frac{2}{\pi} \int_{0}^{\infty} Q[S(Q)-1] \sin (Q r) d Q
$$

Where $r$ is the radial distance.

In order to compare or to fit the experimental $\mathrm{G}(r)$ against a structural model, the $\mathrm{G}(r)_{\text {calc }}$ can be calculated from a set of structural parameters according to: Neutron scattering length $b(\mathrm{fm})$, atomic number $Z$ and ionic radius for $\mathrm{La}, \mathrm{Ni}$ and $\mathrm{O}$.

$$
G(r)_{\mathrm{calc}}=\frac{1}{r} \sum_{\nu} \sum_{\mu}\left[\frac{b_{\nu} b_{\mu}}{\bar{b}^{2}} \delta\left(r-r_{v \mu}\right)\right]-4 \pi r \rho_{0}
$$

where $v$ and $\mu$ are the ions involved, $b$ is the coherence scattering length and $\rho_{0}$ is the number density. The function $\mathrm{G}(r)$ indicates the probability of finding a couple of atoms at a certain $r$-value with an integrated intensity dependent on the coherence scattering lengths $b$ of the elements involved (see Eq. (2)).

Aim of this study is to provide a crystallographic description of the deviations with respect to its average periodic structure of $\mathrm{La}_{2} \mathrm{NiO}_{4+\delta}$ at high temperature.

We performed X-Rays (XRPD) and Neutron Powder Diffraction (NPD) experiments since both techniques can be considered complementary probes. In fact, X-rays scattering lengths are proportional to the atomic number, while neutrons scattering lengths $b$ just depend on the single isotopes. In particular, considering a natural isotopic compositions, the neutrons $b$ values for $\mathrm{La}$, Ni and $\mathrm{O}$ are 8.24, 10.3 and $5.803 \AA$ respectively. For that reason, neutrons are more sensitive to oxygen related parameters than X-rays.

\section{Experimental}

High quality single crystals of $\mathrm{La}_{2} \mathrm{NiO}_{4+\delta}$ were grown by the floating zone technique described elsewhere [11]. Typically rods of about 4 to $8 \mathrm{~mm}$ diameter and several centimeters long could be obtained. The as grown single crystals were post annealed under oxygen at $380^{\circ} \mathrm{C}$, yielding a stoichiometric of $\mathrm{La}_{2} \mathrm{NiO}_{4.18}$. The as obtained crystals were crashed and ground into a pestle-mortar in order to produce a fine powder. Such powdered sample was the subject of synchrotron X-Ray (XRPD) and Neutron Powder Diffraction (NPD) measurements performed at the D4c diffractometer (NPD) [12] of the ILL (Grenoble, France) at room temperature and at the ID15b [13] and at the ID11 [14] beam lines (XRPD) of the ESRF (Grenoble, France) respectively at room temperature and as a function of temperature (high temperature, reaching about $450^{\circ} \mathrm{C}$ ).

NPD pattern was collected at room temperature at the D4c instrument at ILL with an incident neutron wavelength $\lambda=0.4989 \AA$, covering a $Q$ range between 0.35 and $23.6 \AA^{-1}$. The powdered sample was loaded into a $5 \mathrm{~mm}$ diameter vanadium can (net sample quantity was a mass $\mathrm{m}=5.6848 \mathrm{~g}$ ), 

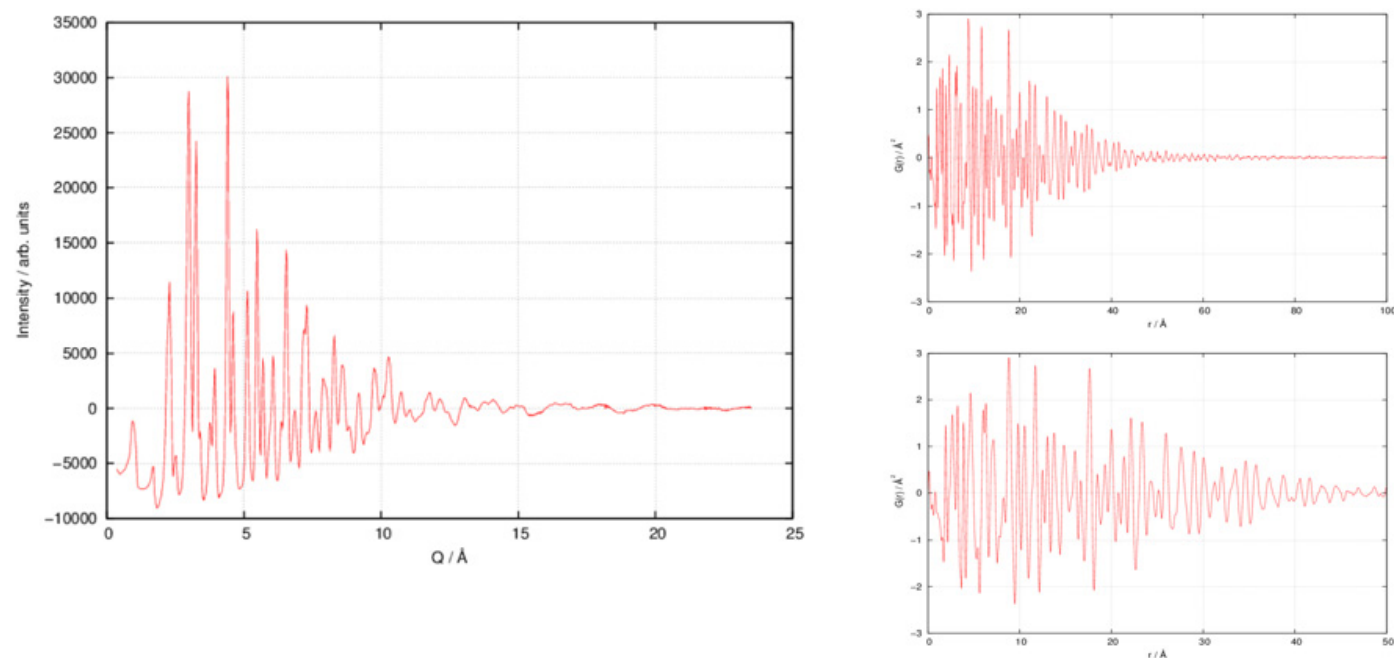

Figure 2. NPD pattern of $\mathrm{La}_{2} \mathrm{NiO}_{4+\delta}$ recorded on D4c, after appropriate background subtraction, absorption and incoherent-scattering contribution corrections (on the left). On the right, pair distribution function $\mathrm{G}(r)$ obtained from the NPD D4c data after appropriate background subtraction, absorption and incoherent-scattering contribution corrections of the neutron powder diffraction pattern. Two different real space ranges are showed: up to $100 \AA$ (left) and up to $20 \AA$ (right).

which was placed into an evacuated bell jar (to reduce air scattering with neutrons). Empty bell jar, vanadium bar, empty can and a boron powder have been measured for about one hour in order to properly subtract the background. The backgrounds were measured periodically during the experiment in order to check their stability. Raw neutron data were collected for about 1.5 hours and processed by regrouping repeated scans of the D4c detectors over the available range of scattering angles in the reciprocal space. Appropriate background (a linear combination of the vanadium sample holder and empty bell jar contribution) was subtracted from the raw data, taking into account absorption effects [15] as well as the incoherent-scattering contribution. A standard multiple-scattering [16] and Placzeck [17] corrections were applied. As an example, Fig. 2 shows the NPD pattern of $\mathrm{La}_{2} \mathrm{NiO}_{4+\delta}$ as obtained on D4c, as well as the corresponding pair distribution function G(r). Fourier transform was performed using a software procedure developed at D4c.

XRPD data were collected in the EH1 of the ID11 beam line of the ESRF, using a Frelon camera, and a Linkam oven system to warm up the sample up to a maximum of $\sim 450{ }^{\circ} \mathrm{C}$. Sample to detector distance was $140 \mathrm{~mm}$, and it was further calibrated. Sample was ground to a finer powder and loaded into a $0.5 \mathrm{~mm}$ diameter quartz capillary. The capillary was placed horizontally in the Linkam oven. Experimental data were collected at incident X-ray energy $E=97 \mathrm{keV}$, corresponding to a wavelength $\lambda=0.12782(1) \AA$, up to $Q_{\max }=26.0 \AA^{-1} .202 \mathrm{D}$ images of 15 seconds each were collected at room temperature, at $\mathrm{T}=450{ }^{\circ} \mathrm{C}$ and again at room temperature to check for reproducibility. Azimuthal integration of raw images was performed using software package $p y F A I$ [18]. X-rays real space data were computed using the PDFgetX3 software [19]: data were corrected for diffraction from the container, sample self-absorption, multiple scattering, Laue diffuse and Compton scattering. An example of $P D F g e t X 3$ configuration file is given in the Annexe.

Figure 3 shows the G(r) obtained from the ID11 data at room temperature and at $450{ }^{\circ} \mathrm{C}$. As an example for a PDFgetX3 configuration file is given in the Annexe. 

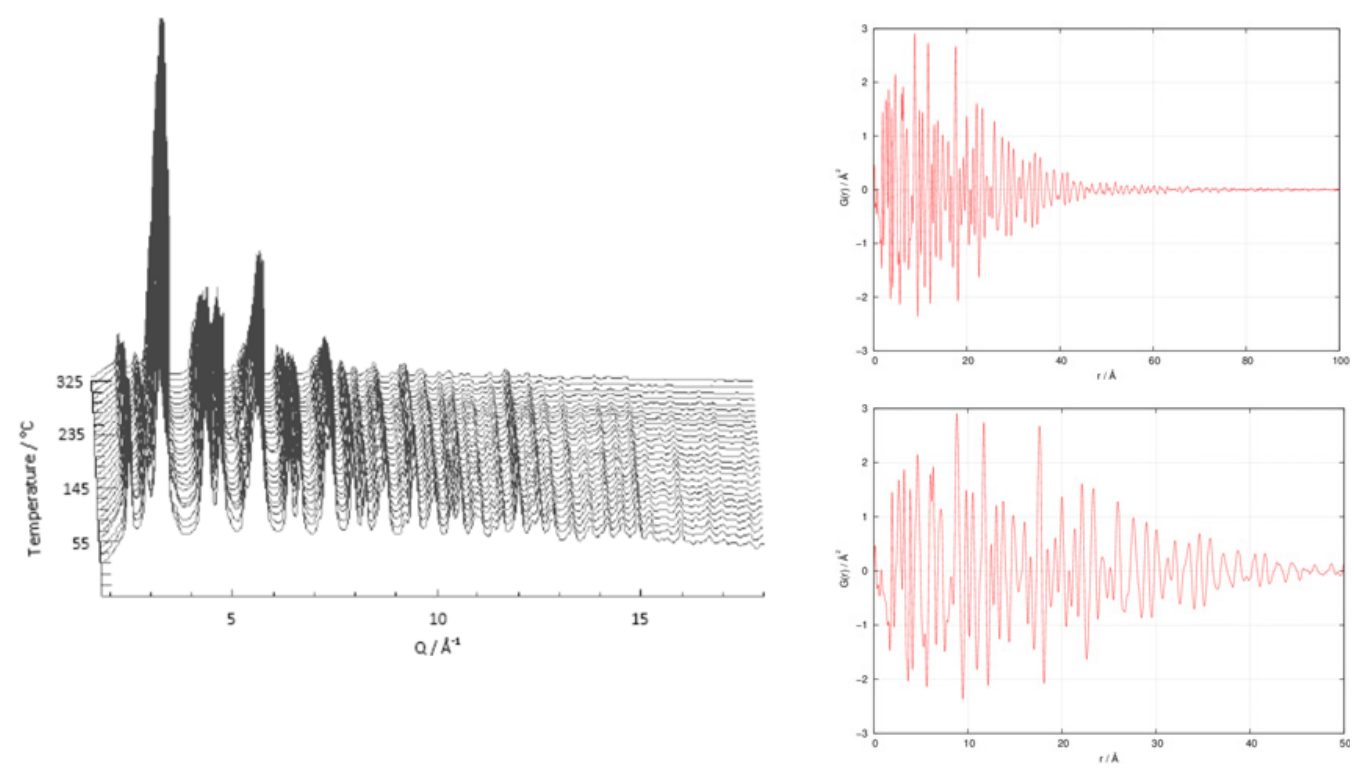

Figure 3. In-situ XRPD patterns of $\mathrm{La}_{2} \mathrm{NiO}_{4+\delta}$ in the $(0,15) \AA^{-1}$ range recorded on ID11 on warming from RT to about $325^{\circ} \mathrm{C}$ (on the left). On the fight, the corresponding pair distribution functions $\mathrm{G}(\mathrm{r})$ obtained from the X-ray powder diffraction, at room temperature. Two different real space ranges are showed: up to $100 \AA$ (left) and up to $20 \AA$ (right).

\section{Results}

In order to study the structural disorder induced in $\mathrm{La}_{2} \mathrm{NiO}_{4+\delta}$ by the insertion of interstitial oxygen atoms, an analysis in real space is performed, which means that PDF data are refined by against a structural model by minimizing the difference via a non-linear least square procedure. This is done employing the PDFGui software [20]. The agreement between model and experimental data is given by the $\mathrm{R}_{\mathrm{w}}$ factor:

$$
R_{W}=\left(\frac{\sum_{i=1}^{n} \omega\left(r_{i}\right)\left[G_{o b s}\left(r_{i}\right)-G_{c a l c}\left(r_{i}\right)\right]^{2}}{\sum_{i=1}^{n} \omega\left(r_{i}\right) G_{o b s}^{2}\left(r_{i}\right)}\right)^{\frac{1}{2}}
$$

where $\omega\left(r_{i}\right)=1 / \sigma^{2}\left(r_{i}\right)$ and $\sigma\left(r_{i}\right)$ is the standard deviation at a distance $r_{i}$.

One of the main issues of PDF modelling concerns the necessity of attaining the best possible agreement between calculated and experimental data, still recurring to a limited amount of parameters in order to guarantee statistical significance, thus avoiding the overparametrization of the fit. In the context of PDF analysis, the latter turns out to depend on the number of refined parameters, the interatomic distance range as well as the maximum value of wavevector $Q$ available. In the present study, $\mathrm{Q}_{\max }$ values of $23.6 \AA^{-1}$ and $26.0 \AA^{-1}$ were employed for NPD and XRPD data, respectively.

In order to model the experimental data by using a reduced number of parameters, thus enhancing statistical significance, we have first described the unit cell starting from a simplified version of $o i$-LNO, that is by neglecting any disordering on the apical oxygen site, still retaining the $14 / \mathrm{mmm}$ tetragonal structure: this structural model is here defined as O3ap-LNO. Note that the model does account for the intercalation of interstitial oxygen in the form of partial occupation of the $\mathrm{O} 2$ site. The fit of the O3ap-LNO model employing spherical displacement parameters is reported in the panel (a) of Fig. 4. The main atom pairs corresponding to each peak are indicated in the same figure. The fit quality is not 


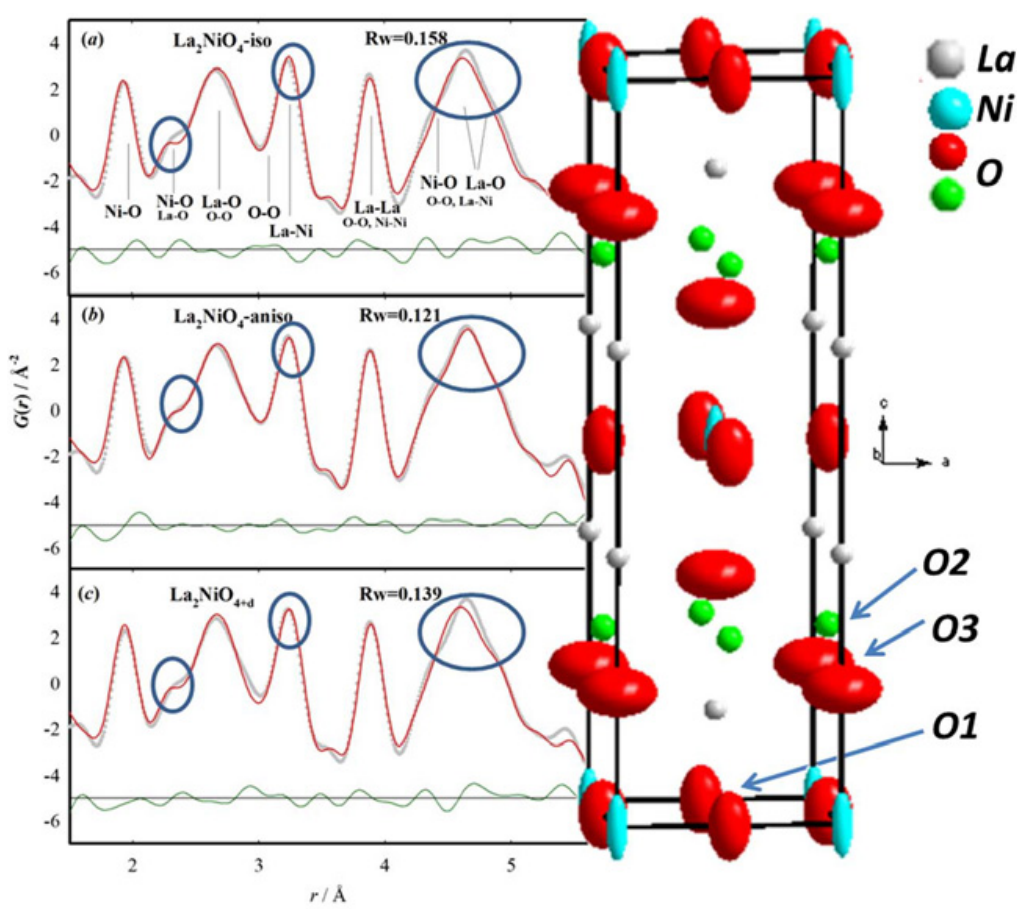

Figure 4. Right-hand panel: fit of the $\mathrm{La}_{2} \mathrm{NiO}_{4}$ structure using NPD data (room temperature) after the O3apLNO model with isotropic ADP (a, top), using the same model with anisotropic atomic displacement parameters (b, middle) and using the oxygen intercalated (oi-LNO) model (c, bottom), respectively. Empty blue circles: experimental data; red solid lines: calculated profile; difference curves shown below in green. On the right-hand panel, the ellipsoids related to ADP are graphically displayed.

poor, yet some features (see blue circles) still need to be fixed. Information about possible deviations from the stoichiometric atomic arrangement was sought for by accounting for non-spherical thermal displacements. The latter, indeed, highlights possible configurations of static disorder. The fit, reported in the panel (b) of Fig. 4, shows improvements of some features (see circles) related mainly to metaloxygen and metal-metal connections. Moreover, the overall residual parameter $\mathrm{R}_{\mathrm{w}}$ decreases from 0.158 to 0.121 . No noteworthy correlations were returned by minimization procedure. Ellipsoids related to the above anisotropic displacement parameters (ADP) are graphically displayed in the right-hand panel of Fig. 4. The in-plane disorder of the apical oxygen tallies with the site split described by the oxygen intercalated model. In addition, out-of-plane displacements were noticed for the $\mathrm{O}$ site on the basal plane as well as for the Ni ions. On the opposite, La site seems not to be affected by relevant disorder. Note that very similar features were observed in ref. [8] by single crystal investigation. In order to confirm the displacement of the apical oxygen site, the $o i$-LNO model was refined against the same experimental NPD data. The model was meant to get rid of ADP, giving instead a more explicit description of the atomic positions than the O3ap-LNO with ADP. With reference to the in- plane disorder of the apical oxygen site, refinements of PDF data were carried out in the $1.5<\mathrm{r}<5.6 \AA$ range by recursively shifting by 0.01 step size the $x$ coordinate of the $\mathrm{O} 4$ site, which corresponds to the actual displacement from the ideal apical $\mathrm{O} 3$ site. The evolution of the fit residual against the magnitude of $x(\mathrm{O} 4)$ is displayed in Fig. 5. The trend clearly evidences two minima, corresponding to two different atomic configurations. The best fit is reported in panel (c) of Fig. 4. The fit doesn't match the quality reached by employing 


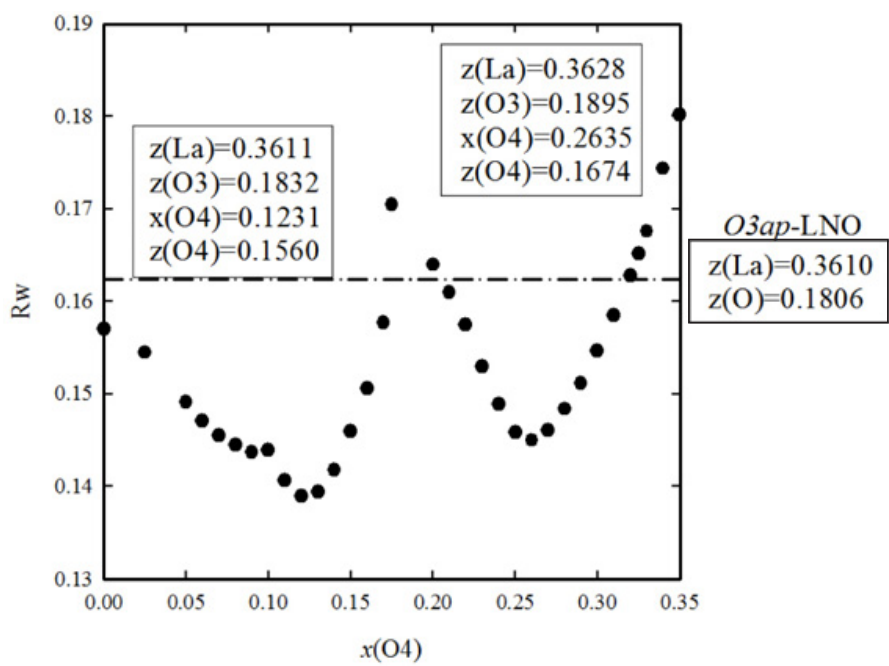

Figure 5. The residual $\mathrm{R}_{\mathrm{w}}$ factor of the fit computed up to $5.6 \AA$ as a function of the $x$ coordinate of the $\mathrm{O} 4$ site. Dashed line represents the residual $R_{w}$, in the same range, for the O3ap model, which neglects the apical O4 split site.

Table 3. Structural data of $\mathrm{La}_{2} \mathrm{NiO}_{4.18}$ refined against NPD and XRPD PDF data at room temperatures.

\begin{tabular}{|l|l|l|}
\hline & NPD & XRPD \\
\hline $\mathrm{a} / \AA$ & $3.854(4)$ & $3.868(9)$ \\
\hline $\mathrm{c} / \AA$ & $12.84(4)$ & $12.71(5)$ \\
\hline $\mathrm{z}(\mathrm{La})$ & $0.3619(5)$ & $0.3615(8)$ \\
\hline $\mathrm{z}(\mathrm{O})$ & $0.1793(10)$ & $0.197(13)$ \\
\hline $\mathrm{U} 11 \equiv \mathrm{U} 22(\mathrm{La}) / \AA^{2}$ & $0.0037(15)$ & $0.0041(15)$ \\
\hline $\mathrm{U} 33(\mathrm{La}) / \AA^{2}$ & $0.0020(11)$ & $0.0040(18)$ \\
\hline $\mathrm{U} 11 \equiv \mathrm{U} 22(\mathrm{Ni}) / \AA^{2}$ & $0.0021(11)$ & $0.0057(23)$ \\
\hline $\mathrm{U} 33(\mathrm{Ni}) / \AA^{2}$ & $0.030(4)$ & $0.0043(35)$ \\
\hline $\mathrm{U} 11 \equiv \mathrm{U} 22(\mathrm{O} 1) / \AA^{2}$ & $0.018(3)$ & $0.08(5)$ \\
\hline $\mathrm{U} 33(\mathrm{O} 1) / \AA^{2}$ & $0.012(3)$ & $0.007(30)$ \\
\hline $\mathrm{U} 11(\mathrm{O} 3) / \AA^{2}$ & $0.032(6)$ & $0.031(30)$ \\
\hline $\mathrm{U} 22(\mathrm{O} 3) / \AA^{2}$ & $0.045(6)$ & $0.13(10)$ \\
\hline $\mathrm{U} 33(\mathrm{O} 3) / \AA^{2}$ & $0.013(3)$ & $0.027(9)$ \\
\hline
\end{tabular}

ADP, yet one should consider that the present model does not account for any displacement of Ni nor of basal $\mathrm{O}$ ions, which were, though, probed by the analysis of ADP.

Similar results are obtained by PDF analysis of X-ray data collected at room temperature. The corresponding structural parameters are given in Table 3. In this case, though, the absolute displacements values have to be handled with care, being X-ray data mostly sensitive to La-involving atom pairs, whist their precision on the O-related parameters is quite low. Nevertheless, the in-plane disordering of the apical $\mathrm{O}$ site is apparent even from $\mathrm{X}$-ray data, further supporting the split of the apical site of the $\mathrm{NiO}_{6}$ octahedra.

\section{Discussion}

In Fig. 6, a comparison by overlaying the room temperature and the high temperature X-ray PDF data is shown: we observe major changes with regards to the cation sites, especially for La-La and Ni distances 


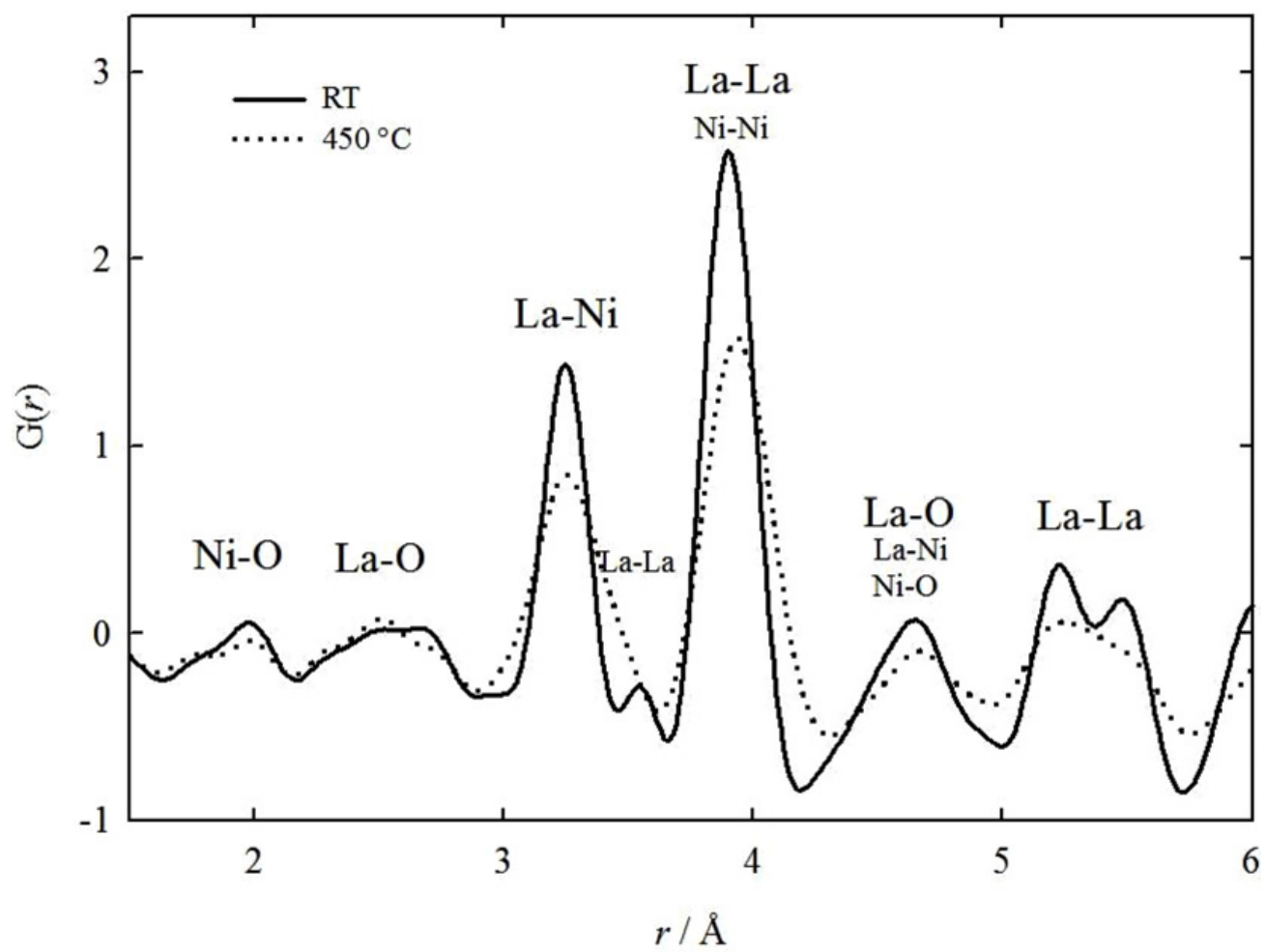

Figure 6. Comparison between the room temperature (solid line) and the high temperature $\left(450^{\circ} \mathrm{C}\right.$, dashed line) $\mathrm{X}$-ray PDF data, in the low-rrange.

(the peaks become non-Gaussian), thus suggesting an enhancement of the disorder described above, because of a larger distribution of interatomic distances. In order to obtain the scattering densities of the apical oxygen atoms, neutron single diffraction was carried out on the $5 \mathrm{C} 2$ diffractometer installed at the ORPHEE reactor at $\lambda=0.83 \AA$. Data collection was undertaken up to $2 \Theta=115^{\circ}$, equivalent to $(\sin \Theta / \lambda) \max =1.02^{-1}$. No absorption correction was applied, as the linear absorption coefficient is small with $\mu=0.1 \mathrm{~cm}^{-1}$ at $\lambda=0.83 \AA$. Data collection of half of the Ewald-sphere was undertaken and subsequent structure refinement carried out using SHELXL. The Maximum Entropy Algorithm was then applied to the obtained phased reflection set using the MEED program [21]. Figure 7 shows the reconstructed scattering densities of the apical oxygen plane at $\mathrm{z}=0.174$ for different temperatures. At low temperatures like $20 \mathrm{~K}$, oxygen must be considered not to be mobile any more. At this temperature we observe for the apical oxygen atoms, in addition to a broad maximum, four discrete maxima with almost no overlap to the central peak. The four maxima reflect the symmetrical increase of the $\mathrm{O}_{\text {int }}\left(\mathrm{O}_{\mathrm{ap}}\right)_{4}$ tetrahedron (which is the $\mathrm{O} 2(\mathrm{O} 3)_{4}$ tetrahedron, with the notation adopted in this work, see Table 1) and increasing the distances for $\mathrm{O}_{\text {int }}-\mathrm{O}_{\text {ap }}$ to about $2.7 \AA$ as reported in [8].

With increasing temperature complete overlap between the central and outer positions are already observed at ambient, indication the activation of libration modes along [110], i.e. the presence of large amplitudes towards the directions of the interstitial sites. These dynamically amplified displacements must be considered as a key issue to realize how oxygen hopping can be attained in the lower temperature regime down to ambient. They thus indicate a dynamic exchange between apical and interstitial lattice 
$20 \mathrm{~K}$ :

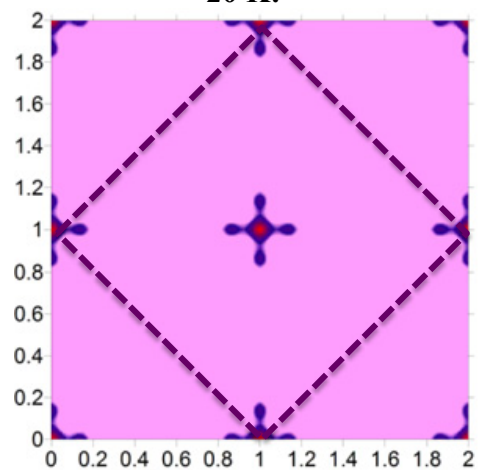

$293 \mathrm{~K}$ :

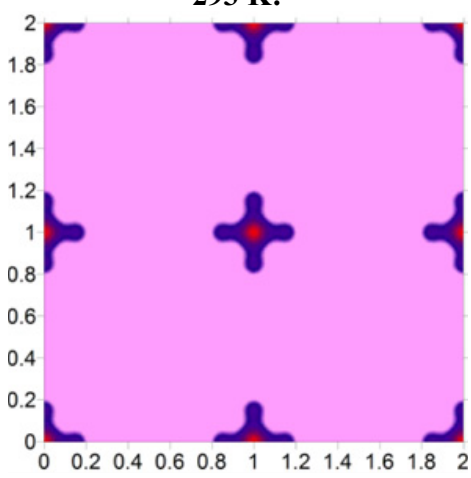

$673 \mathrm{~K}$ :

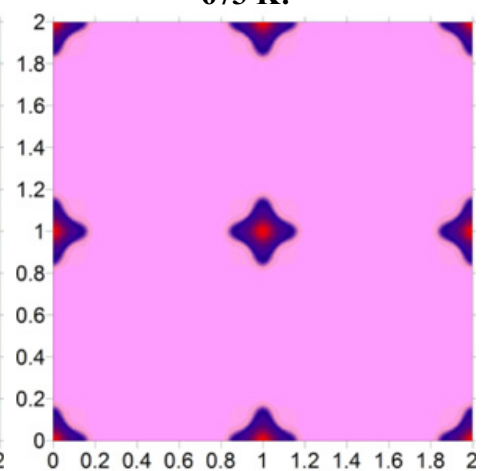

Figure 7. Maximum Entropy reconstruction of the apical-O plane of $\mathrm{La}_{2} \mathrm{NiO}_{4.18}$ at different temperatures at $\mathrm{z}=0.17$, obtained from single crystal neutron diffraction up to $1.05 \AA^{-1}$ (in $\sin \Theta / \lambda$ ), showing selectively the scattering density of the apical oxygen atoms. A red dashed line outlines the F-cell. The additional maxima around the central peak at $20 \mathrm{~K}$ pointing towards the [110] direction of the F-centred unit cell, are a consequence of the repulsive interaction between the interstitial and apical atoms. While the latter are pushed away from their equilibrium position, the corresponding $\mathrm{O}_{\mathrm{ap}}-\mathrm{O}_{\text {int }}$ distances increase to about $2.7 \AA$, while $2.15 \AA$ are obtained when considering their average positions. At already ambient temperature the distinct maxima transform towards a dynamic $\mathrm{O}_{\text {ap }}$ disorder scenario, resulting in strong anharmonic displacement factors for the apical oxygen atoms, which by symmetry impose to constrain $\mathrm{U}_{11}=\mathrm{U}_{2} 2$. Large anisotropic amplitudes of $\mathrm{O}_{\text {ap }}$ displacements towards [110] naturally favour oxygen diffusion towards the interstitial lattice sites in $(1 / 4,1 / 4,1 / 4)$.

sites, allowing a 1D shallow potential diffusion pathway along [110]. The necessity of interstitial oxygen atoms for low temperature oxygen mobility, realized by phonon assisted diffusion mechanism becomes thus clear as they induce these strong distortions of the apical oxygen atoms, facilitating them to jump towards the interstitial sites and vice-versa. This goes in the same direction when increasing the temperature to $673 \mathrm{~K}$, where dynamical displacements are already highly activated. The density cards especially of the apical oxygen atoms are thus of utmost importance for a microscopic understanding of the diffusion mechanism.

Compared with MEM, which gives the most probable reconstruction of the nuclear density, PDF analysis is able to give direct observation of the shift of the apical oxygen atoms and the consequent increase of the tetrahedral sites for oxygen intercalated phase. Coupling the neutron probe using the NPD data from D4c allows us to study O-O distances which are less precisely detectable through $\mathrm{X}$-Rays and to prove this shift.

\section{Conclusions and outlook}

In ref. [8] it was observed that, apposite to what one would expect, thermal factor of the interstitial oxygen atoms decreases by increasing temperature, while that of the apical oxygen atoms does increase with temperature. Here, an experimental approach involving total X-Rays and Neutrons Powder Diffraction and Pair Distribution Function analysis was performed in order to investigate the disorder involving apical and the interstitial oxygen atoms in the structure of $\mathrm{La}_{2} \mathrm{NiO}_{4+\delta}$ at room and at high temperature. This has demonstrated the in-plane disordering of the apical $\mathrm{O}$ site, supporting the split of the apical site of the $\mathrm{NiO}$ octahedra.

As further investigation, a study of the thermal parameters of both interstitial and apical could be used to investigate the disorder in the structure induced by the presence of the extra interstitial oxygen 
atom. When disorder is present, atoms occupying the same site in different cell can have equilibrium positions, which differ from the average one adding a contribution to the thermal parameter often defined as static contribution. Such an experimentally observed shift could be then considered a clear fingerprint of such static disorder.

The authors acknowledge the European Synchrotron Radiation Facility and the Institut Laue Langevin for provision of beam time; they also wish to thank Dr. G.B.M Vaughan for assistance in using the ID11 beam line, Dr. H.E. Fischer for assistance in using the D4c instrument and Dr. M. Coduri for useful discussion.

\section{References}

[1] Wattiaux, A.; Park, J. C.; Grenier, J. C.; Pouchard, M. Comptes Rendus de l'Academie des Sciences, Serie 2 1990, 310, 1047.

[2] Perrichon, A.; Piovano, A.; Boehm, M.; Zbiri, M.; Johnson, M.; Schober, H.; Ceretti, M.; Paulus, W. J. Phys. Chem. C 2014, 119, 1557.

[3] Villesuzanne, A.; Paulus, W.; Cousson, A.; Hosoya, S.; Le Dréau, L.; Hernandez, O.; Prestipino, C.; Ikbel Houchati, M.; Schefer, J. Journal of Solid State Electrochemistry 2011, 15, 357.

[4] Gauquelin, N.; Weirich, T.; Ceretti, M.; Paulus, W.; Schroeder, M. Monatshefte für Chemie / Chemical Monthly 2009, 140, 1095.

[5] Bassat, J.-M.; Burriel, M.; Wahyudi, O.; Castaing, R.; Ceretti, M.; Veber, P.; Weill, I.; Villesuzanne, A.; Grenier, J.-C.; Paulus, W.; Kilner, J. A. The Journal of Physical Chemistry C 2013.

[6] Chroneos, A.; Parfitt, D.; Kilner, J. A.; Grimes, R. W. Journal of Materials Chemistry 2010, 20, 266.

[7] Chroneos, A.; Parfitt, D.; Kilner, J. A.; Grimes, R. W. J. Mater. Chem. 2009, 20, 266.

[8] Paulus, W.; Cousson, A.; Dhalenne, G.; Berthon, J.; Revcolevschi, A.; Hosoya, S.; Treutmann, W.; Heger, G.; Le Toquin, R. Solid State Sciences 2002, 4, 565.

[9] Billinge, S. J. L.; Kanatzidis, M. G. Chemical Communications 2004, 749.

[10] Egami, T. Journal of Physics and Chemistry of Solids 1995, 56, 1407.

[11] Ammerahl, U.; Dhalenne, G.; Revcolevschi, A.; Berthon, J.; Moudden, H. Journal of Crystal Growth 1998, 193, 55.

[12] Fischer, H. E.; Cuello, G. J.; Palleau, P.; Feltin, D.; Barnes, A. C.; Badyal, Y. S.; Simonson, J. M. Appl Phys A 2002, 74, s160.

[13] http://www.esrf.eu/UsersAndScience/Experiments/StructMaterials/ID15.

[14] http://www.esrf.eu/UsersAndScience/Experiments/StructMaterials/ID11.

[15] Paalman, H. H.; Pings, C. J. Journal of Applied Physics 1962, 33, 2635.

[16] Blech, I. A.; Averbach, B. L. Physical Review 1965, 137, A1113.

[17] Yarnell, J. L.; Katz, M. J.; Wenzel, R. G.; Koenig, S. H. Physical Review A 1973, 7, 2130.

[18] Ashiotis, G.; Deschildre, A.; Nawaz, Z.; Wright, J. P.; Karkoulis, D.; Picca, F. E.; Kieffer, J. Journal of Applied Crystallography 2015, 48, 510.

[19] Juhas, P.; Davis, T.; Farrow, C. L.; Billinge, S. J. L. Journal of Applied Crystallography 2013, 46, 560.

[20] Farrow, C. L.; Juhas, P.; Liu, J. W.; Bryndin, D.; Božin, E. S.; Bloch, J.; Proffen, T.; Billinge, S. J. L. Journal of Physics: Condensed Matter 2007, 19, 335219.

[21] Kumazawa, S.; Kubota, Y.; Takata, M.; Sakata, M.; Ishibashi, Y. Journal of Applied Crystallography 1993, 26, 453. 


\section{EPJ Web of Conferences}

\section{Annexes}

\section{PDFgetX3 configuration file}

X-rays real space data were computed using the PDFgetX3 software [19]: data were corrected for diffraction from the container, sample self-absorption, multiple scattering, Laue diffuse and Compton scattering using the following configuration file (e.g. ID15b data):

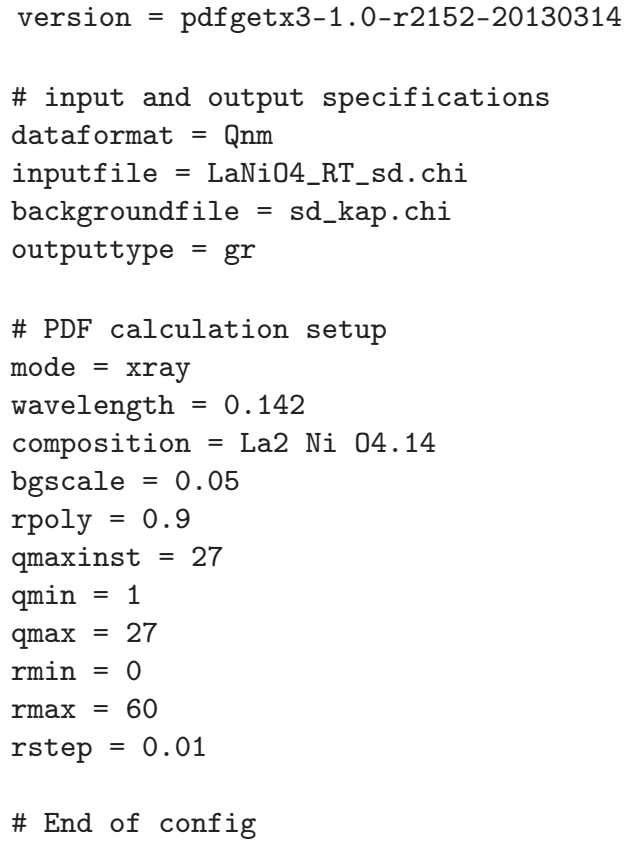

